\title{
Complete pathological response in patients with locally advanced cancer of breast and positive Her2/neu with neoadjuvant chemotherapy Heidy Cedeño*1, Rafael Araúz ${ }^{3}$, Fernando Cebamanos² ${ }^{2}$ Idalmis de Torraza ${ }^{4}$ and Ana Porcell 4
}

\author{
Address: ${ }^{1}$ Department Internal Medicine. Instituto Oncológico Nacional, Rep de Panama, ${ }^{2}$ Department of Medical Oncology. Instituto \\ Oncológico Nacional, Rep de Panama, ${ }^{3}$ Department of Radiation Oncology Instituto Oncológico Nacional, Rep de Panama and ${ }^{4}$ Department of \\ Pathology. Instituto Oncológico Nacional, Rep de Panama \\ Email: Heidy Cedeño* - drayramos@hotmail.com \\ * Corresponding author
}

from 24th Annual Meeting of the National Cancer Institute of Mexico Mexico City, Mexico. 14-17 February 2007

Published: 5 February 2007

BMC Cancer 2007, 7(SuppI I):A50 doi:I0.I I86/I47I-2407-7-SI-A50

This article is available from: http://www.biomedcentral.com/I47I-2407/7/SI/A50

(C) 2007 Cedeño et al; licensee BioMed Central Ltd.

\section{Background}

This retrospective study was made with the purpose of evaluating the rate of complete pathological response (CPR) of the tumor and CPR of the axillary lymph nodes in patients with Her2/neu dealt with preoperative systemic treatment.

\section{Materials and methods}

We retrospectively reviewed thirty-three (n: 33) clinical records of females with diagnosis of locally advanced breast cancer with positive Her 2/neu receptors, which was treated with preoperative chemotherapy in the Institute from January 2004 to December 2005. The median of age was of $58+/-14$ years (rank: $36-91$ years). The initial clinical stages were: IIA, n: 1 (3\%); IIB, n: 3 (9\%), IIIA, n: $19(58 \%)$ and IIIB, n: $10(30 \%)$. The initial nodal stage were: N0, n: $5(15 \%)$; N1, n: $9(27 \%)$, and N2, n: 19 (58\%). Twenty-one (64\%) patients had both hormonal ER and PR negatives. The combination of Anthracyclines and Taxanes was prescribed in n: $17(52 \%)$ and $n: 8$ $(24 \%)$ received in addition neoadjuvant Trastuzumab (Herceptin). A breast conservative surgery was performed in four (n: 4) patients, the rest of patients a modified radical mastectomy was made. The median number of dissected nodes was of $13+/-7$ nodes (rank: 3-32) and median of the percentage of metastasic nodes was of $15+$ / - 24\% (rank: 0-72\%).

\section{Results}

The rate of tumor CPR was achieved in $9 / 33$ patients $(27 \%)$ and the rate of nodal CPR was noted in $11 / 33$ patients (33\%). The patients who received neoadjuvant Trastuzumab had same tumor CPR and axillary lymph node CPR of 38\% (3/8). An 18\% (6/33) of the patients presented total CPR (tumor and nodal). The tumor or axillary lymph nodes CPR was no associated with age, histology, menopause status, initial clinical or nodal stage, use of neoadjuvant Trastuzumab or the scheme of chemotherapy or the state of ER/PR. Sixty-six percent of complete pathologic responders had negative axillary lymph nodes compared to $21 \%$ of patients who did not have a complete pathologic response $(\mathrm{P}=0.013)$.

\section{Conclusion}

The rate of tumor and nodal CPR is within of the reported in the literature. It is important to emphasize the promising benefit and the controversial role of preoperative Trastuzumab in these patients. Others molecular biological factors must be studied like favorable predicting of pathological response. 